\title{
COVID-19 Hospitalization by Race and Ethnicity: Association with Chronic Conditions Among Medicare Beneficiaries, January 1-September 30, 2020
}

\author{
Man-Huei Chang ${ }^{1} \cdot$ Ramal Moonesinghe ${ }^{2} \cdot$ Benedict I. Truman ${ }^{1}$ \\ Received: 4 November 2020 / Revised: 29 December 2020 / Accepted: 29 December 2020 / Published online: 8 January 2021 \\ (C) This is a U.S. government work and not under copyright protection in the U.S.; foreign copyright protection may apply 2021
}

\begin{abstract}
Objectives We assessed the association between hospitalization for illness from COVID-19 infection and chronic conditions among Medicare beneficiaries (MBs) with fee-for-service (FFS) claims by race and ethnicity for January 1-September 30, 2020. Methods We used 2020 monthly Medicare data from January 1-September 30, 2020, reported to the Centers for Medicare and Medicaid Services to compute hospitalization rates per 100 COVID-19 MBs with FFS claims who were hospitalized (ICD-10CM codes: B97.29 before April 1, 2020; ICD-10-CM codes: U07.1 from April 1, 2020, onward) with or without selected chronic conditions. We used logistic regression to estimate adjusted odds ratios with $95 \%$ confidence intervals for association of personlevel rate of being hospitalized with COVID-19 and each of 27 chronic conditions by race/ethnicity, controlling for age, sex, and urban-rural residence among MBs.

Results COVID-19-related hospitalizations were associated with all selected chronic conditions, except osteoporosis and Alzheimer disease/dementia among COVID-19 MBs. The top five conditions with the highest odds for hospitalization among COVID-19 MBs were end-stage renal disease (adjusted odds ratios (aOR): 2.15; 95\% CI: 2.10-2.21), chronic kidney disease (aOR: 1.54; 95\% CI: 1.52-1.56), acute myocardial infarction (aOR: 1.45; 95\% CI: 1.39-1.53), heart failure (aOR: 1.43; 95\% CI: 1.41-1.44), and diabetes (aOR: 1.37; 95\% CI: 1.36-1.39).

Conclusions Racial/ethnic disparities in hospitalization rate persist among MBs with COVID-19, and associations of COVID-19 hospitalization with chronic conditions differ among racial/ethnic groups in the USA. These findings indicate the need for interventions in racial/ethnic populations at the highest risk of being hospitalized with COVID-19.
\end{abstract}

Keywords Coronavirus $\cdot$ Multiple chronic conditions $\cdot$ Medicare $\cdot$ Health status disparities $\cdot$ Race factors $\cdot$ Ethnic groups

\section{Introduction}

The number of cases of SARS CoV-2 infection, the virus that causes COVID-19 illness, has rapidly increased in the USA. Since the first case of patient with COVID-19 was reported in January 2020 [1], approximately 9.3 million cases and > 232,000 confirmed COVID-19-associated deaths have been reported in the USA as of November 3, 2020 [2]. Studies have

Man-Huei Chang

mdc9@cdc.gov

1 Office of the Director, National Center for HIV/AIDS, Viral Hepatitis, STD, and TB Prevention, Centers for Disease Control and Prevention (CDC), 1600 Clifton Rd., NE, Mail Stop US 8-6, Atlanta, GA 30329-4027, USA

2 Office of Minority Health and Health Equity, CDC, Atlanta, GA, USA reported that COVID-19-associated hospitalizations and mortality in the USA were higher among older adults, males, and Black persons [3, 4]. Risk factors associated with hospital admission include comorbidity, public insurance, residence in a low-income area, and obesity [4]. Persons in any age group with certain underlying medical conditions have been reported to be at increased risk for severe illness from COVID-19; these medical conditions include cancer, chronic kidney disease, chronic obstructive pulmonary disease (COPD), immunocompromised state from solid organ transplant, obesity, serious heart conditions, sickle cell disease, and type 2 diabetes mellitus [5-7]. Approximately $90 \%$ of persons hospitalized for severe illness from COVID-19 have been observed to have one or more of these underlying medical conditions [3].

Black persons and other racial and ethnic minority groups with COVID-19 also are more likely to have one or more medical conditions than White patients with COVID-19 [8, 
9]. Racial and ethnic disparities in COVID-19 hospitalization and mortality have been observed, with higher rates among Black persons, Hispanic persons, and American Indian/ Alaska Native (AIs/ANs) persons, compared with White persons $[8,10]$. Although associations of hospitalization with chronic conditions have been reported and concerns regarding racial and ethnic disparities with COVID-19 burden on disproportionately affected populations have been discussed, the association between hospitalized versus nonhospitalized COVID-19 patients with or without chronic conditions has not been well evaluated. Prior studies of COVID-19 hospitalizations did not collect data regarding nonhospitalized patients and did not compare the prevalence of underlying chronic conditions between hospitalized and nonhospitalized patients with COVID-19 [3]. Additionally, assessments have been limited to small samples in certain geographic areas [11]. Therefore, the difference in characteristics between hospitalized and nonhospitalized patients with COVID-19 and the impact of associated chronic conditions has not been assessed in large-scale samples. Limited information is available regarding risk factors, such as social determinants of health, urban versus rural residence, and chronic conditions associated with hospitalization among patients with COVID-19, especially among older adults in different racial and ethnic groups who are likely to have a higher prevalence of certain chronic conditions. Older persons typically have a greater disease and economic burden of disease than younger persons [12]. Medicare claims data for older persons and those with a disability or with end-stage renal disease offer an opportunity for examining the association of racial and ethnic disparities among hospitalized COVID-19 patients with chronic conditions. To fill the knowledge gap, we assessed if Medicare beneficiaries (MBs) with fee-for-service (FFS) claims for COVID-19, who also had a chronic health condition, are more likely to receive hospital inpatient services for COVID-19associated outcomes than COVID-19 MBs without chronic conditions. We also assessed racial and ethnic disparities in hospitalizations among MBs with COVID-19 and chronic conditions.

\section{Study Data and Methods}

\section{Sources and Populations}

We used data from the 2020 preliminary monthly Medicare with FFS claims for January 1-September 30, 2020, available through the Centers for Medicare and Medicaid Services (CMS, https://www.cms.gov/) Virtual Research Data Center to calculate hospitalization rates for COVID-19-associated outcomes among MBs. A Medicare COVID-19 confirmed case was defined as an $\mathrm{MB}$ with a primary or secondary International Classification of Diseases Clinical Modification
(10 rev.) (ICD-10-CM) [13] diagnosis code of B97.29 (other coronavirus as the cause of diseases classified elsewhere) on an FFS claim for January 1-March 31, 2020, or diagnosis code U07.1 (2019 novel coronavirus, COVID-19, confirmed by laboratory testing) from April 1, 2020, onward for any health care setting. A Medicare COVID-19-hospitalized beneficiary was defined as an MB with a diagnosis of COVID-19 on an FFS claim for an inpatient hospital setting.

We classified each COVID case as yes/no for beneficiaries with or without a claim for hospitalization. We estimated frequencies of COVID patients with or without hospitalization by sex (male or female), race/ethnicity (non-Hispanic White [NHW], non-Hispanic Black [NHB], Hispanic, Asian/Pacific Islander [A/PI], American Indian/Alaska Native [AI/AN], or other/unknown), US Census region of residence (Northeast, West, Midwest, or South), county-level of urban-rural residence, Medicare entitlement (end-stage renal disease, disabled, or aged), and Medicare eligibility status (Medicare only or dual Medicare and Medicaid). We classified the county-level six National Center for Health Statistics (Centers for Disease Control and Prevention) urban-rural classification scheme [14] to the following three urban-rural groups: urban (large central metropolitan and large fringe metropolitan), sub-urban (medium metropolitan and small metropolitan), and rural (micropolitan and noncore). We included 27 chronic health, mental health, and substance abuse conditions as follows: acute myocardial infarction; atrial fibrillation; heart failure; hypertension; ischemic heart disease; stroke; peripheral vascular disease; chronic kidney disease; diabetes; hyperlipidemia; anemia; diabetes; Alzheimer disease; depression; obesity; COPD; schizophrenia; lung cancer; liver disease; viral hepatitis; HIV/AIDS; asthma; osteoporosis; tobacco use; opioid use disorder; spina bifida and other congenital anomalies of the nervous system; and sickle cell disease. We also classified cardiovascular disease, including acute myocardial infarction, atrial fibrillation, heart failure, hypertension, ischemic heart disease, stroke, and peripheral vascular disease. These conditions were defined by using the 2018 (the most recently/updated available data) Chronic Conditions Data Warehouse predefined algorithms [15]. These conditions were selected because of (a) high prevalence among MBs or (b) COVID-19 patients with these conditions likely being at higher risk for hospitalization than those without these conditions. We summarized the number of comorbidities for each beneficiary with or without hospitalization as four categories: $0,1-3,4-5$, or $\geq 6$.

\section{Analysis}

First, we calculated the following two measures:

A. Hospitalization rates for COVID-19 per $100 \mathrm{MBs}$ with COVID-19: The number of MBs with COVID-19 who were hospitalized divided by the total number of MBs 
with confirmed COVID-19 and Medicare FFS claims, multiplied by 100 .

B. Hospitalization rate per 100,000 MBs: The number of Medicare COVID-19 hospitalized beneficiaries divided by the total number of beneficiaries enrolled in Medicare FFS, multiplied by 100,000.

Second, we analyzed the characteristics of FFS MBs with confirmed COVID-19 and with or without COVID-19-related hospitalizations including sex, race and ethnicity, US Census region, urban-rural residence, Medicare entitlement, Medicare eligibility status, and comorbidity. We also examined the differences in characteristics between hospitalized and nonhospitalized COVID-19 MBs in a 2-tailed chi-square test at a significance level of $P<.05$. Third, we estimated the prevalence of chronic conditions among MBs with a confirmed COVID-19 illness and who had versus who did not have a hospitalization claim. Lastly, we used multivariable logistic regression to examine the association between hospitalization and the selected chronic conditions among patients with COVID-19 by race/ethnicity. We estimated adjusted odds ratios (aORs) with 95\% confidence intervals (95\% CIs) for COVID-19 hospitalization among those COVID-19 MBs with each chronic condition versus those COVID-19 MBs without that chronic condition, adjusting for age, sex, and urban-rural residence. We used a 2-tailed Wald chi-square test for statistical significance at $P<.05$ for differences in the rate of hospitalized beneficiaries with COVID-19 and with a chronic condition versus those COVID-19 MBs without that chronic condition. All analyses were performed by using SAS® 9.4 and SAS Enterprise Guide ${ }^{\circledR} 7.1$ (SAS Institute, Inc., Cary, NC) in the secured environment of the CMS Virtual Research Data Center through the Chronic Conditions Data Warehouse [16]. Federal officials not involved in the study approved the ethical conduct of the analysis and reporting of deidentified Medicare data that did not involve human research.

\section{Results}

Of 39.3 million MBs with FFS claims during January 1September 30, 2020, the total number of MBs with COVID19 was 710,980; among those, 207,600 COVID-19-related hospitalizations occurred as of September 30, 2020. The hospitalization rate for COVID-19 was 527.9/100,000 MBs (Table 1). The hospitalization rate per 100 COVID-19 MBs was 29.20 for all racial/ethnic groups. By race and ethnicity, $\mathrm{NHB}$ and $\mathrm{AI} / \mathrm{AN}$ MBs had higher hospitalization rates for COVID-19 at 1299.4 and 1170.6 hospitalizations/100,000 MBs, respectively, than other racial and ethnic groups. Compared with non-Hispanic White (NHW) MBs (396.1/ 100,000 enrolled MBs), hospitalization rates were $\geq 1.2$ times as high for non-Hispanic Black (NHB) (3.3), American Indian/Alaska Native (AI/AN) (3.0), Hispanic (2.6), and Asian/Pacific Islander (A/PI) (1.2). Hospitalization rate related to COVID-19 per 100 COVID-19 MBs was highest for COVID-19 MBs who are AI/AN (44.97), followed by NHB (40.63), Hispanic (35.96), A/PI (30.94), and NHW (25.22). Among patients with COVID-19, 1 in $2 \mathrm{AI} / \mathrm{AN}$ or NHB MBs and 1 in 4 NHW MBs were admitted to the hospital for COVID-19 illness.

Hospitalized COVID-19 MBs were more likely than those nonhospitalized COVID-19 MBs to be aged $\geq 75$ years ( $52.5 \%$ versus $48.3 \%$ ), to be male ( $50.0 \%$ versus $41.4 \%)$, to be NHB (22.3\% versus $13.4 \%)$, to be residing in the South United States ( $42.6 \%$ versus $37.7 \%$ ), to be residing in an urban county ( $60.6 \%$ versus $59.0 \%)$, to have end-stage renal disease (7.9\% versus 3.2\%), to be enrolled in both Medicare and Medicaid (64.8\% versus $47.7 \%$ ), and to have multiple chronic conditions (46.4\% versus $36.9 \%$ with six or more selected conditions, Table 2). Supplemental Table 1 presents the prevalence of chronic conditions among MBs with a confirmed COVID-19 illness with hospitalization versus without hospitalization. The 10 chronic conditions ranked for highest
Table 1 Hospitalization rates per 100,000 Medicare beneficiaries (MBs) with fee-for-service (FFS) claims, by race/ethnicity USA, January 1-September 30, 2020

\begin{tabular}{llllll}
\hline Race/ethnicity & $\begin{array}{l}\text { No. of } \\
\text { MBs }\end{array}$ & $\begin{array}{l}\text { No. of } \\
\text { COVID- } \\
\text { 19 MBs }\end{array}$ & $\begin{array}{l}\text { No. of } \\
\text { hospitalized } \\
\text { COVID-19 MBs }\end{array}$ & $\begin{array}{l}\text { Hospitalization } \\
\text { rate per 100,000 } \\
\text { MBs }\end{array}$ & $\begin{array}{l}\text { Hospitalization rate } \\
\text { per 100 COVID-19 } \\
\text { MBs }\end{array}$ \\
\hline $\begin{array}{l}\text { Non-Hispanic } \\
\text { White }\end{array}$ & $30,271,436$ & 475,368 & 119,898 & 396.1 & 25.22 \\
$\begin{array}{c}\text { Non-Hispanic } \\
\text { Black }\end{array}$ & $3,558,790$ & 113,822 & 46,242 & 1299.4 & 40.63 \\
$\begin{array}{c}\text { Hispanic } \\
\text { Asian/Pacific }\end{array}$ & $2,779,571$ & 79,842 & 28,713 & 1033.0 & 35.96 \\
$\quad \begin{array}{l}\text { Islander } \\
\text { American } \\
\text { Indian/Alas- } \\
\text { ka Native }\end{array}$ & 212,108 & 5521 & 2483 & 485.8 & 30.94 \\
$\begin{array}{l}\text { Other/unknown } \\
\text { Total }\end{array}$ & $1,227,958$ & 16,355 & 4053 & 1170.6 & 44.97 \\
\hline
\end{tabular}


Table 2 Baseline characteristics of Medicare beneficiaries (MBs) with fee-for-service claims for coronavirus disease 2019 (COVID-19), by hospitalization status, USA, January 1September 30, 2020

\begin{tabular}{|c|c|c|c|}
\hline Characteristics & $\begin{array}{l}\text { Hospitalized } \\
100 \%(n=207,600)\end{array}$ & $\begin{array}{l}\text { Nonhospitalized } \\
100 \%(n=503,380)\end{array}$ & $P$ value \\
\hline Age (yrs) & & & $<.0001 *$ \\
\hline$\leq 64$ & 15.3 & 14.0 & - \\
\hline $65-74$ & 32.2 & 37.7 & - \\
\hline$\geq 75$ & 52.5 & 48.3 & - \\
\hline Sex, $\%$ & & & $<.0001 *$ \\
\hline Male & 50.0 & 41.4 & - \\
\hline Female & 50.0 & 58.6 & - \\
\hline Race/ethnicity, \% & & & $<.0001 *$ \\
\hline Non-Hispanic White & 57.8 & 70.6 & - \\
\hline Non-Hispanic Black & 22.3 & 13.4 & - \\
\hline Hispanic & 13.8 & 10.2 & - \\
\hline Asian/Pacific Islander & 3.0 & 2.8 & - \\
\hline American Indian/Alaska Native & 1.2 & 0.6 & - \\
\hline Other/unknown & 2.0 & 2.4 & - \\
\hline US Census region, by state, $\%$ & & & $<.0001 *$ \\
\hline Northeast & 26.5 & 38.9 & - \\
\hline Midwest & 17.1 & 15.3 & - \\
\hline South & 42.6 & 37.7 & - \\
\hline West & 13.7 & 17.7 & - \\
\hline US territories & 0.2 & 0.5 & - \\
\hline Urban-rural classification & & & $<.0001 *$ \\
\hline Urban & 60.6 & 59.0 & - \\
\hline Sub-urban & 25.9 & 26.8 & - \\
\hline Rural & 13.5 & 14.2 & - \\
\hline Medicare entitlement & & & $<.0001 *$ \\
\hline End-stage renal disease & 7.9 & 3.2 & - \\
\hline Disabled & 12.1 & 13.0 & - \\
\hline Aged & 79.9 & 83.8 & - \\
\hline Medicare eligibility status & & & $<.0001 *$ \\
\hline Medicare only & 35.2 & 52.3 & - \\
\hline Dual Medicare and Medicaid & 64.8 & 47.7 & - \\
\hline Number of comorbidities ${ }^{\mathrm{a}}$ & & & $<.0001 *$ \\
\hline 0 & 15.0 & 17.2 & - \\
\hline $1-3$ & 19.9 & 25.6 & - \\
\hline $4-5$ & 18.8 & 20.4 & - \\
\hline$\geq 6$ & 46.4 & 36.9 & - \\
\hline
\end{tabular}

*MBs with COVID-19 with fee-for-service claims were significantly different from nonhospitalized MBs with COVID-19 by chi-square test at $P<.05$

${ }^{a}$ Includes acute myocardial infarction, atrial fibrillation, heart failure, hypertension, ischemic heart disease, stroke, peripheral vascular disease, chronic kidney disease, diabetes, hyperlipidemia, anemia, diabetes, Alzheimer disease, depression, obesity, COPD, schizophrenia, lung cancer, liver disease, viral hepatitis, HIV/AIDS, asthma, ESRD, osteoporosis, tobacco use, opioid use disorder, spina bifida and other congenital anomalies of the nervous system, and sickle cell disease prevalence among MBs hospitalized with COVID-19 were cardiovascular disease $(78.39 \%)$, hypertension $(71.42 \%)$, hyperlipidemia $(54.21 \%)$, diabetes $(45.27 \%)$, chronic kidney disease $(43.61 \%)$, anemia $(40.60 \%)$, ischemic heart disease $(40.30 \%)$, rheumatoid arthritis/osteoarthritis $(40.22 \%)$, depression (28.91\%), and heart failure (28.1\%; Fig. 1). Prevalence rates of chronic conditions among MBs hospitalized with COVID-19 were significantly different from those without hospitalization $(P<.05)$, except depression. The largest gaps in prevalence for three chronic conditions between 


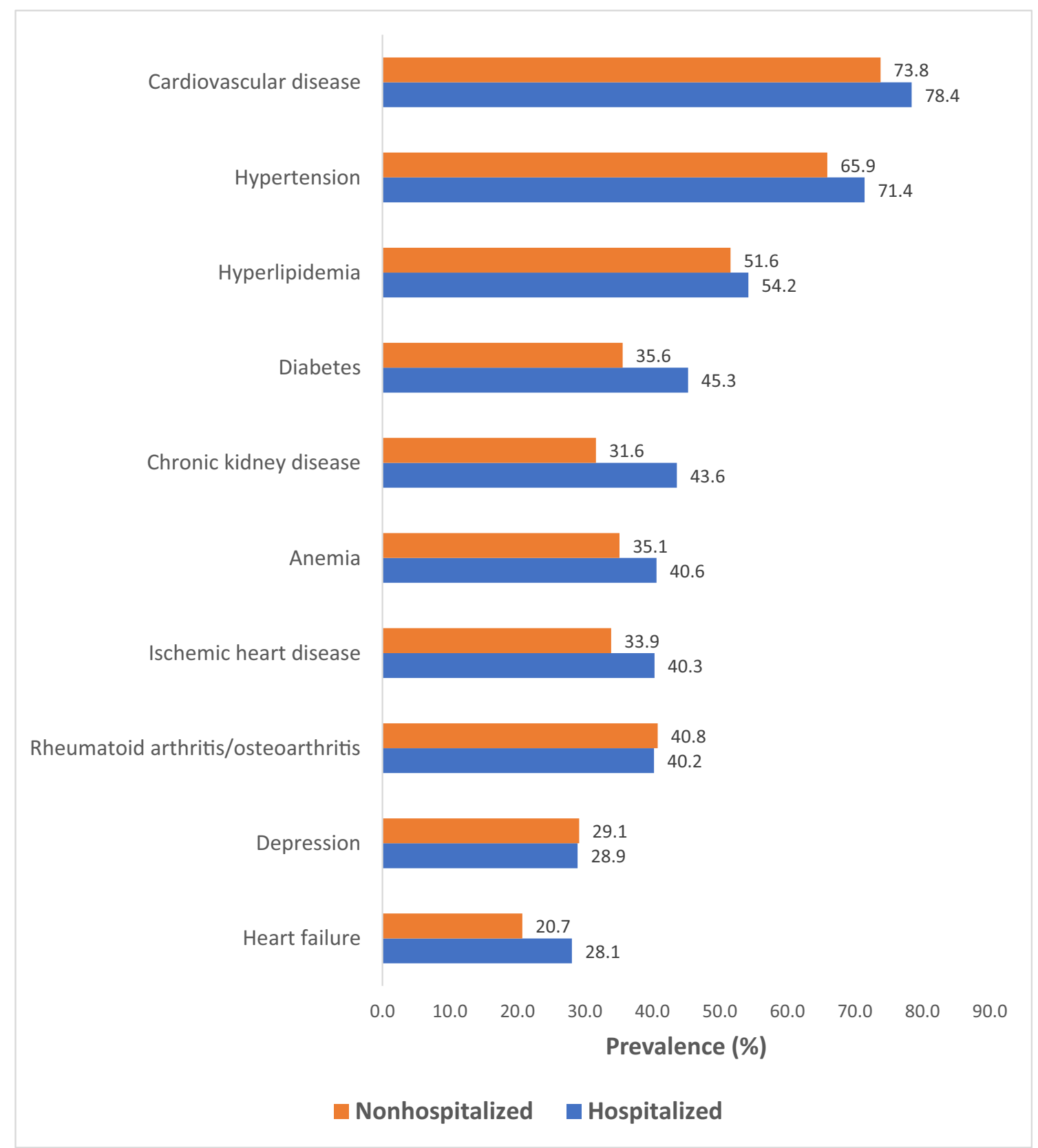

Fig. 1 Ten chronic conditions ${ }^{\mathrm{a}, *}$ ranked for highest prevalence among Medicare beneficiaries with coronavirus disease 2019 (COVID-19), by hospitalization status, USA, January 1-September 30, 20,200. ${ }^{a}$ Cardiovascular disease includes acute myocardial infarction; atrial fibrillation; heart failure; hypertension; ischemic heart disease; stroke/transient

MBs with COVID-19 infection who were hospitalized versus nonhospitalized were observed for chronic kidney disease (11.98\%), diabetes $(9.68 \%)$, and heart failure $(7.35 \%)$.

Table 3 presents the aORs and $95 \%$ CIs of selected chronic conditions associated with COVID-19-related hospitalizations among beneficiaries with a confirmed COVID19 illness. A statistically significant association $(P<.0001)$ of all selected chronic conditions, except osteoporosis and Alzheimer disease/dementia, and COVID-19-related ischemic attack; and peripheral vascular disease. *Significantly difference in prevalence of all chronic conditions, except depression, between hospitalized and nonhospitalized MBs with COVID-19 for chi-square test at $P<.05$

hospitalization among overall MBs with COVID-19 was observed after controlling for age, sex, race and ethnicity, and urban-rural residence. The top five conditions with the highest odds for hospitalization among MBs with COVID19 were end-stage renal disease (aOR: 2.15 ; $95 \%$ CI: 2.10 2.21 ), chronic kidney disease (aOR: 1.54 ; $95 \%$ CI: 1.52 1.56), acute myocardial infarction (aOR: $1.45 ; 95 \% \mathrm{CI}$ : 1.39-1.53), heart failure (aOR: 1.43 ; 95\% CI: 1.41-1.44), and diabetes (aOR: 1.37 ; 95\% CI: 1.36-1.39). Additional 
Table 3 Adjusted $^{\mathrm{a}}$ odds ratios (aORs) and 95\% confidence intervals (CIs) for association of selected chronic conditions and COVID-19-related hospitalizations among Medicare beneficiaries (MBs) with a confirmed COVID-19 illness, USA, January 1-September 30, 2020

\begin{tabular}{|c|c|}
\hline Condition & $\mathrm{aOR}(95 \% \mathrm{CI})$ \\
\hline End-stage renal disease & $2.15(2.10-2.21)$ \\
\hline Chronic kidney disease & $1.54(1.52-1.56)$ \\
\hline Acute myocardial infarction & $1.45(1.39-1.53)$ \\
\hline Heart failure & $1.43(1.41-1.44)$ \\
\hline Diabetes & $1.37(1.36-1.39)$ \\
\hline Chronic obstructive pulmonary disease & $1.36(1.34-1.38)$ \\
\hline Obesity & $1.36(1.34-1.37)$ \\
\hline Spina bifida and other congenital anomalies of the nervous system & $1.21(1.10-1.35)$ \\
\hline Lung cancer & $1.21(1.15-1.28)$ \\
\hline Opioid use disorder & $1.19(1.15-1.23)$ \\
\hline Ischemic heart disease & $1.27(1.25-1.28)$ \\
\hline Hepatitis & $1.15(1.11-1.20)$ \\
\hline Schizophrenia & $1.10(1.08-1.13)$ \\
\hline Hypertension & $1.24(1.22-1.25)$ \\
\hline Liver disease & $1.18(1.15-1.21)$ \\
\hline Atrial fibrillation & $1.18(1.16-1.20)$ \\
\hline Cardiovascular disease $^{\mathrm{b}}$ & $1.24(1.23-1.26)$ \\
\hline Anemia & $1.21(1.20-1.22)$ \\
\hline Asthma & $1.13(1.11-1.16)$ \\
\hline Tobacco & $1.03(1.01-1.05)$ \\
\hline Peripheral vascular disease & $1.19(1.17-1.20)$ \\
\hline Sickle cell disease & $1.55(1.31-1.82)$ \\
\hline Stroke & $1.13(1.11-1.15)$ \\
\hline HIV/AIDS & $0.88(0.82-0.94)$ \\
\hline Hyperlipidemia & $1.12(1.11-1.14)$ \\
\hline Depression & $1.07(1.06-1.08)$ \\
\hline Rheumatoid arthritis/osteoarthritis & $1.03(1.02-1.04)$ \\
\hline Osteoporosis* & $1.00(0.98-1.02)$ \\
\hline Alzheimer disease/dementia* & $1.01(0.99-1.03)$ \\
\hline
\end{tabular}

associated chronic conditions also included COPD, obesity, spina bifida and other congenital anomalies of the nervous system, lung cancer, opioid use disorder, ischemic heart disease, hepatitis, schizophrenia, hypertension, liver disease, atrial fibrillation, cardiovascular disease, anemia, asthma, tobacco, peripheral vascular disease, sickle cell disease, stroke, HIV/AIDS, hyperlipidemia, and depression. Supplemental Table 2 presents aORs and 95\% CIs for hospitalization among COVID-19 MBs with chronic conditions versus those COVID-19 MBs without chronic conditions, by racial and ethnic groups, including NHW, NHB, Hispanic, and A/PIs. AI/ANs were not included in the analysis because of insufficient samples. Increased COVID-19related hospitalization was significantly $(P<.05)$ associated with end-stage renal disease, chronic kidney disease, heart failure, obesity, acute myocardial infarction, ischemic heart disease, anemia, peripheral vascular disease, atrial fibrillation, hyperlipidemia, stroke, and Alzheimer disease/ dementia among COVID-19 MBs in the four racial and ethnic groups. However, COPD, schizophrenia, cardiovascular disease, and tobacco were associated with COVID-19related hospitalization among $\mathrm{NHW}, \mathrm{NHB}$, and Hispanic COVID-19 MBs. This association was not observed among COVID-19 AI/PI MBs. For lung cancer, the association with increased COVID-19 hospitalization was observed only among COVID-19 NHW MBs. On the other hand, osteoporosis was not found to be associated with COVID-19 hospitalization among any racial/ethnic group. Racial/ ethnic disparities in COVID-19-associated hospitalizations were observed among MBs with selected chronic conditions 
Table 4 Top ${ }^{\mathrm{a}}$ five chronic conditions associated with hospitalization for COVID-19 illness, among Medicare beneficiaries (MBs) with a confirmed COVID-19 illness, by race and ethnicity ${ }^{\mathrm{b}}$, USA, January 1-September 30, 2020

\begin{tabular}{lll}
\hline Race/ethnicity & Condition* & aOR (95\% CI) \\
\hline Non-Hispanic White & End-stage renal disease & $2.28(2.18-2.38)$ \\
& Chronic kidney disease & $1.53(1.51-1.55)$ \\
& Chronic obstructive pulmonary disease & $1.47(1.45-1.50)$ \\
Non-Hispanic Black & Heart failure & $1.45(1.43-1.48)$ \\
& Acute myocardial infarction & $1.43(1.34-1.52)$ \\
& End-stage renal disease & $1.97(1.89-2.04)$ \\
& Sickle cell disease & $1.63(1.36-1.95)$ \\
& Chronic kidney disease & $1.56(1.52-1.60)$ \\
Hispanic & Acute myocardial infarction & $1.42(1.27-1.58)$ \\
& Heart failure & $1.36(1.32-1.40)$ \\
& End-stage renal disease & $2.45(2.33-2.58)$ \\
& Chronic kidney disease & $1.56(1.51-1.61)$ \\
& Acute myocardial infarction & $1.52(1.32-1.76)$ \\
& Heart failure & $1.41(1.36-1.47)$ \\
& Diabetes & $1.34(1.30-1.38)$ \\
& End-stage renal disease & $1.98(1.76-2.23)$ \\
& Acute myocardial infarction & $1.90(1.41-2.56)$ \\
& Chronic kidney disease & $1.37(1.28-1.46)$ \\
& Heart failure & $1.31(1.21-1.41)$ \\
& Obesity & $1.24(1.12-1.38)$ \\
& &
\end{tabular}

$a O R$, adjusted (for age, sex, and urban-rural residence) odds ratio

${ }^{\text {a }}$ Ranked by aORs

${ }^{\mathrm{b}}$ American Indian/Alaska Natives are excluded because of insufficient samples

*All top five chronic conditions were significantly associated with COVID-19-related hospitalization for Wald chi-square test at $P<.05$ and the associated effects on conditions varied by racial and ethnic groups. Table 4 includes the top five conditions (highest aORs) associated with hospitalization for COVID-19 illness among MBs who were infected with SARS CoV-2, by race/ethnicity. Racial and ethnic disparities in hospitalization for COVID-19 were observed among MBs with selected conditions. NHW MBs with COVID-19 and having one of any of the selected chronic conditions, including end-stage renal disease, chronic kidney disease, COPD, heart failure, and acute myocardial infarction were more likely (ORs $=1.43-2.28)$ to be admitted to the hospital, compared with those without the conditions. NHB beneficiaries with sickle cell disease (aOR: 1.63; 95\% CI: 1.361.95 ) were more likely to be admitted to the hospital versus those who did not have this condition. Hispanic MBs with diabetes had a 1.34 -time odds (95\% CI: $1.30-1.38$ ); A/PI MBs with obesity had 1.24-time odds (95\% CI: 1.12-1.38), respectively, of being admitted to the hospital than those without diabetes and obesity, respectively. MBs with COVID-19 and having end-stage renal disease were at increased risk to be admitted to hospital among all racial and ethnic groups, with the highest odds (aOR: $2.28 ; 95 \% \mathrm{CI}$ : 2.18-2.38) observed among NHW MBs.

\section{Discussion}

Our findings indicate that racial and ethnic disparities exist in hospitalization rates among enrolled MBs and in MBs with COVID-19. Racial and ethnic minorities (e.g., NHBs and AI/ ANs) were more likely to be hospitalized than NHWs. Especially, AI/AN MBs with COVID-19 were more likely to require inpatient medical services than patients in any other racial and ethnic groups after becoming infected. Our findings also indicate that substantial variation exists between hospitalized and nonhospitalized MBs with COVID-19 by demographic attributes, including age, sex, race/ethnicity, geographic region, urbanization, Medicare entitlement, Medicare, and Medicaid dual-eligibility status, and comorbidity. Hospitalized MBs with COVID-19 were more likely to be aged $\geq 75$ years, to be male, to be Black, to reside in the northeast US region, to reside in urban counties, to have end-stage renal disease, to be enrolled in both Medicare and Medicaid programs, and to have multiple chronic conditions.

Social determinants of health are known to play a principal role in disease outcomes and have a substantial effect on life expectancy [17]. They also seem to have a considerable effect on COVID-19 morbidity and mortality and hospitalizations 
for COVID-19-associated outcomes [18, 19]. Inequities in the social determinants of health that disproportionately affect racial and ethnic minorities include poverty, lack of health care access to testing centers and medical facilities, being uninsured, using public transportation, employees in jobs that require physical contact, or living in crowded spaces and housing environments [20, 21]. A prior study reported that the COVID-19 illness rate is three times higher among predominantly Black counties than among predominantly White counties in the USA [22]. Similarly, our results also found that the COVID-19-associated hospitalizations for NHB MBs were 3 times or higher than NHW MBs. Further studies regarding social inequalities in health are needed to quantify the effect on COVID-19 patients among all disproportionately affected populations in different age and racial/ethnic groups.

Our results support prior analyses [3-7] in that they demonstrate a statistically significant association $(P<.0001)$ between hospitalizations attributable to COVID-19 illness and selected chronic conditions, including end-stage renal disease, chronic kidney disease, cardiovascular disease, COPD, diabetes, obesity, hypertension, and lung cancer. Medicare MBs infected with COVID-19, who have one or more of the selected chronic conditions, were at increased risk for being admitted to the hospital for illness from COVID-19 compared with those without these chronic conditions. We also observed that the statistical significance of associations of hospitalization and these chronic conditions varied slightly among the different racial and ethnic groups. For example, end-stage renal disease, heart-related conditions, COPD, lung cancer, opioid use disorder, stroke, and spina bifida and other congenital anomalies of the nervous system were significantly associated with hospitalizations among NHW MBs with COVID-19; sickle cell disease among NHB MBs; hepatitis among Hispanic MBs; and obesity among A/PI MBs. Although the magnitude of the risk varied by chronic condition and by race and ethnicity, COVID-19 MBs with one of these chronic conditions were at increased risk to be hospitalized for severe outcomes from COVID-19 than those without. The existing and persistent racial and ethnic disparities among US communities have posed considerable challenges for public health interventions during the US COVID-19 pandemic in 2020.

Our results indicate that the prevalence of such conditions was higher among hospitalized MBs compared with nonhospitalized patients (e.g., chronic kidney disease, diabetes, heart failure, ischemic heart disease, and hypertension). These selected chronic conditions were more prevalent (e.g., a difference of $11.98 \%$ for chronic kidney disease) among hospitalized versus nonhospitalized MBs with COVID-19. Our analysis represents estimates for the Medicare population with a large proportion of older adults; thus, prevalence of chronic conditions in our study might be higher than it is among the general population.
For example, a comprehensive systematic review using meta-analyses reported approximately 16\%, 12\%, 8\%, and $8 \%$ prevalence rates among hospitalized patients for hypertension, cardiovascular disease, smoking history, and diabetes, respectively [23]. A recent CDC report from COVID-NET data stated that approximately $90 \%$ of persons hospitalized have one or more underlying medical conditions [3].

\section{Limitations}

Our findings are subject to several limitations. First, these results were based on MBs who had claims for COVID-19 illness and claims for hospitalization from COVID-19 illness. We were unable to obtain characteristics of MBs who were asymptomatic, were false positive, and might not have a claim for COVID-19 in the Medicare data system. Second, our data are claims-based, and the population is restricted to MBs with FFS claims covered by Medicare insurance; claims were submitted by physicians' offices, inpatient hospitals, or laboratories. Our estimates cannot account for claims not submitted from health care settings. Additionally, demographic distributions and chronic disease prevalence among the Medicare population are different from the US general population. Therefore, our estimates might not be directly comparable with those in prior studies that used data collected from surveillance systems. Third, this analysis includes preliminary data from the monthly Medicare FFS claims only but did not include Medicare Advantage encounter data because those 2020 data are not yet available. Moreover, these results are based on the preliminary monthly inpatient and outpatient data, which might change as additional data are available. Historically, only approximately $40 \%$ of FFS claims are received by CMS during the first month after the date of service, and $90 \%$ are received within 3 months [24]. Our estimates are likely to represent an underestimate because of the delay in data collection and data reporting. Fourth, chronic condition prevalence in this study was calculated by using conditionspecific variables from the most recent 2018 Chronic Conditions Data Warehouse. Our estimates in chronic condition prevalence might be changed if more current chronic condition data become available. Fifth, we estimated the association of COVID-19 hospitalization with each chronic condition separately but did not adjust for the combined effect of multiple chronic conditions. Sixth, our analysis used ICD-10CM codes defined by CMS [24]. Therefore, our results might not be directly comparable with estimates for cases defined by other sources [25]. Lastly, we assessed hospitalizations among MBs with COVID-19 with chronic conditions by using limited data and information (e.g., basic demographic characteristics) available in Medicare claims data. Other factors contributing to health care usage, racial and ethnic disparities, or possible associated conditions for illness were not considered 
in the analyses (e.g., physician availability, geographical access to services, education, or local policies in preventive measures, including social distancing or wearing face coverings), or other conditions (e.g., pregnancy).

\section{Conclusion}

Our findings confirm the association between hospitalization for COVID-19 illness and chronic conditions among MBs with COVID-19 infection. Racial/ethnic disparities in hospitalization rate persist among MBs with COVID-19, and associations of COVID-19-related hospitalization with chronic conditions differ among racial/ethnic groups in the USA. These findings indicate the need for interventions in racial/ ethnic populations at the highest risk of being hospitalized with COVID-19. The analysis and findings of the study illustrate how Medicare claims data can be used within limitations to supplement data available from notifiable disease surveillance systems and hospital utilization surveys.

Supplementary Information The online version contains supplementary material available at https://doi.org/10.1007/s40615-020-00960-y.

Acknowledgments The authors thank the CMS staff for their data support and disclosure view, staff in the Chronic Conditions Warehouse Help Desk for software and technical support, and staff in the Centers for Disease Control and Prevention's Center for Surveillance, Epidemiology, and Laboratory Services for their administrative support. We extend special thanks to C. Kay Smith, MEd, CDC, for editorial support.

Author Contributions Chang had full access to all of the data in the study and takes responsibility for the integrity of the data and the accuracy of the data analysis.

Study concept and design: Chang, Moonesinghe, and Truman

Acquisition of data: Chang and Truman

Research methods, analysis, and interpretation of data: Chang, Moonesinghe, and Truman

Drafting of the manuscript: Chang, Moonesinghe, and Truman.

Statistical analysis: Chang and Moonesinghe

Critical revision of the manuscript for important intellectual content:

Chang, Moonesinghe, and Truman

Administrative, technical, or material support: Chang and Truman

Study supervision: Truman

\section{Compliance with Ethical Standards}

Conflict of Interest The authors declare that they have no conflict of interest.

Ethics Approval Federal officials not involved in the study approved the ethical conduct of the analysis and reporting of deidentified Medicare data that did not involve human research.

Disclaimer The findings and conclusions in this report are those of the authors and do not necessarily represent the official position of the Centers for Disease Control and Prevention.

\section{References}

1. Guan WJ, Ni Z, Hu Y, Liang W, Ou C, He J. et al; China Medical Treatment Expert Group for Covid-19. Clinical characteristics of coronavirus disease 2019 in China. N Engl J Med. 2020;382(18): 1708-20.

2. Johns Hopkins University \& Medicine. COVID-19 map. Baltimore (MD): Johns Hopkins University; 2020. https://coronavirus.jhu. edu/map.html. Accessed 3 Nov 2020.

3. Garg S, Kim L, Whitaker M, O'Halloran A, Cummings C, Holstein $\mathrm{R}$, et al. Hospitalization rates and characteristics of patients hospitalized with laboratory-confirmed coronavirus disease 2019COVID-NET, 14 states, March 1-30, 2020. MMWR Morb Mortal Wkly Rep. 2020;69(15):458-64.

4. Price-Haywood EG, Burton J, Fort D, Seoane L. Hospitalization and mortality among black patients and white patients with COVID-19. N Engl J Med. 2020;382(26):2534-43.

5. Centers for Disease Control and Prevention (CDC). Coronavirus disease 2019 (COVID-19) - people with certain medical conditions. Atlanta (GA): US Department of Health and Human Services, CDC; 2020. https://www.cdc.gov/coronavirus/2019ncov/need-extra-precautions/people-with-medical-conditions.html. Accessed 3 Nov 2020.

6. Guan WJ, Liang WH, Zhao Y, Liang HR, Chen ZS, Li YM, et al. Comorbidity and its impact on 1590 patients with COVID-19 in China: a nationwide analysis. Eur Respir J. 2020;55(5):2000547.

7. Bolin Wang B, Li R, Lu Z, Huang Y. Does comorbidity increase the risk of patients with COVID-19: evidence from meta-analysis. Aging (Albany NY) Actions. 2020;12(7):6049-57.

8. Hsu HE, Ashe EM, Silverstein M, Hofman M, Lange SJ, Razzaghi $\mathrm{H}$, et al. Race/ethnicity, underlying medical conditions, homelessness, and hospitalization status of adult patients with COVID-19 at an urban safety-net medical center-Boston, Massachusetts, 2020. MMWR Morb Mortal Wkly Rep. 2020;69(27):864-9.

9. Hooper MW, Nápoles AM, Pérez-Stable EJ. COVID-19 and racial/ ethnic disparities. JAMA. 2020;323(24):2466-7.

10. Wortham JM, Lee JT, Althomsons S, Latash J, Davidson A, Guerra $\mathrm{K}$, et al. Characteristics of persons who died with COVID-19United States, February 12-May 18, 2020. MMWR Morb Mortal Wkly Rep. 2020;69(28):923-9.

11. Killerby ME, Link-Gelles R, Haight SC, Schrodt CA, England L, Gomes DJ, et al. Characteristics associated with hospitalization among patients with COVID-19-Metropolitan Atlanta, Georgia, March-April 2020. MMWR Morb Mortal Wkly Rep. 2020;69(25): $790-4$.

12. Centers for Disease Control and Prevention. Older adults. Health People 2020. https://www.healthypeople.gov/2020/topicsobjectives/topic/older-adults. Accessed 3 Nov 2020.

13. Centers for Disease Control and Prevention. International Classification of Diseases, Tenth Revision, Clinical Modification (ICD-10-CM). Published July 17, 2020. https://www.cdc.gov/ nchs/icd/icd $10 \mathrm{~cm} . h \mathrm{htm}$. Accessed 3 Nov 2020.

14. Ingram DD, Franco SJ. 2013 NCHS urban-rural classification scheme for counties. National Center for Health Statistics. Vital Health Stat. 2014;2(166). https://www.cdc.gov/nchs/data/series/ sr_02/sr02_166.pdf. Accessed 7 Jan 2021.

15. Chronic Conditions Data Warehouse. Condition categories. Baltimore (MD): Centers for Medicare and Medicaid Services. https://www.ccwdata.org/web/guest/condition-categories. Accessed 3 Nov 2020.

16. Chronic Conditions Data Warehouse. Data dictionaries [Internet]. Baltimore (MD): Centers for Medicare and Medicaid Services; https://www.ccwdata.org/web/guest/data-dictionaries. Accessed 3 Nov 2020 
17. Singh GK, Daus GP, Allender M, Ramey CT, Martin EK, Perry C, et al. Social determinants of health in the United States: addressing major health inequality trends for the nation, 1935-2016. Int J MCH AIDS. 2017;6(2):139-64.

18. Abrams EM, Szefler SJ. COVID-19 and the impact of social determinants of health. Lancet Respir Med. 2020;8(7):659-61.

19. Shah GH, Shankar P, Schwind JS, Sittaramane V. The detrimental impact of the COVID-19 crisis on health equity and social determinants of health. J Public Health Manag Pract. 2020;26(4):317-9.

20. Tsai J, Wilson M. COVID-19: a potential public health problem for homeless populations. Lancet Public Health. 2020;5:e186-7.

21. Williams D, Cooper L. COVID-19 and health equity - a new kind of "herd immunity.". JAMA. 2020;323(24):2478.

22. Yancy CW. COVID-19 and African Americans. JAMA. 2020;323(19):1891-2.
23. Emami A, Javanmardi F, Pirbonyeh N, Akbari A. Prevalence of underlying diseases in hospitalized patients with COVID-19: a systematic review and meta-analysis. Arch Acad Emerg Med. 2020;8(1):e35.

24. Centers for Medicare \& Medicaid Services (CMS). Preliminary Medicare COVID-19 data snapshot. Baltimore (MD): CMS; 2020. https://www.cms.gov/files/document/medicare-covid-19data-snapshot-fact-sheet.pdf. Accessed 3 Nov 2020.

25. Centers for Disease Control and Prevention. ICD-10-CM official coding and reporting guidelines April 1, 2020 through September 30, 2020. https://www.cdc.gov/nchs/data/icd/COVID-19guidelines-final.pdf. Accessed 3 Nov 2020.

Publisher's Note Springer Nature remains neutral with regard to jurisdictional claims in published maps and institutional affiliations. 